\title{
FAST RESPONSE HOT (111) HGCDTE MWIR DETECTORS
}

\author{
Kacper Grodecki'), Piotr Martyniuk'), Małgorzata Kopytko" ${ }^{1)}$, Andrzej Kowalewski ${ }^{1)}$, \\ Dawid Stępień ${ }^{1,2)}$, Artur Kębłowski²), Adam Piotrowski' ${ }^{2}$, Józef Piotrowski ${ }^{2}$, \\ Waldemar Gawron'), Antoni Rogalski ${ }^{1)}$ \\ 1) Military University of Technology, Institute of Applied Physics, Gen. S. Kaliskiego 2, 00-908 Warsaw, Poland \\ $\triangle$ kacper.grodecki@wat.edu.pl, +48261839374,piotr.martyniuk@wat.edu.pl,malgorzata.kopytko@wat.edu.pl, \\ andrzej.kowalewski@wat.edu.pl,dawid.stepien@wat.edu.pl,antoni.rogalski@wat.edu.pl) \\ 2) Vigo System S.A., Poznańska 129/133, 05-850 Ożarow Mazowiecki, Poland \\ (akeblowski@vigo.com.pl,apiotrowski@vigo.com.pl,jpiotrowski@vigo.com.pl,wgawron@vigo.com.pl)
}

\begin{abstract}
In this work we report simulation and experimental results for an MWIR HgCdTe photodetector designed by computer simulation and fabricated in a joint laboratory run by VIGO Sytems S.A. and Military University of Technology. The device is based on a modified $\mathrm{N}^{+} \mathrm{pP}^{+}$heterostructure grown on 2"., epiready, semi-insulating (100) GaAs substrates in a horizontal MOCVD AIX 200 reactor.

The devices were examined by measurements of spectral and time responses as a function of a bias voltage and operating temperatures. The time response was measured with an Optical Parametric Oscillator (OPO) as the source of $\sim 25$ ps pulses of infrared radiation, tuneable in a 1.55-16 $\mu \mathrm{m}$ spectral range. Two-stage Peltier cooled devices $\left(230 \mathrm{~K}\right.$ ) with a $4.1 \mu \mathrm{m}$ cut-off wavelength were characterized by $1.6 \times 10^{12} \mathrm{~cm} \mathrm{~Hz}^{1 / 2} / \mathrm{W}$ peak detectivity and $<1 \mathrm{~ns}$ time constant for $V>500 \mathrm{mV}$.
\end{abstract}

Keywords: HgCdTe, MWIR, photodetector, response time.

\section{Introduction}

The sensitive and fast response mid-IR photodetectors (MWIR) working at high operating temperature (HOT) are of increasing demand for numerous applications in the infrared technology [1]. But obtaining photodetectors of high detectivity and fast response at the same time stays in contradiction. An example is one of the first HOT detectors with narrow-band gap semiconductors used for uncooled detection of $\mathrm{CO}_{2}$ laser radiation [2]. Much faster can be graphene devices [3]. Unfortunately, the devices based on a high recombination rate suffer from a low responsivity since most of the charge carriers recombine before reaching contacts. At the same time, they are noisy due to a statistical nature of the thermal generation-recombination processes. However, the time response of IR photodetectors can be improved by fast carrier collection $[1,4,5]$. For photodiodes the recombination mechanism is generally important at forward, zero and weak reverse bias when the recombination rate is large. The transport removal of charge carriers becomes the dominating mechanism for reverse bias voltages [4]. This can be easier to achieve with thin absorbers. Unfortunately, thin absorber devices will suffer from a low quantum efficiency. The issue is especially pronounced in detection of longer wavelength radiation, due to decreasing absorption. A possible way to solve the problem is to enhance absorption by the use of multi-junction detectors [6].

Hetero-structural $\mathrm{HgCdTe}$ photodiodes, due to the inherent flexibility of design, are very promising for using in sensitive and broadband HOT detectors [1]. Careful optimization of the devices' architectures makes possible to achieve a near BLIP (Background Limited) performance at a $\mathrm{GHz}$ frequency range. 
In this work we present the theoretical modelling and experimental results for $4.1 \mu \mathrm{m}$ cutoff wavelength photodiodes at $T=230 \mathrm{~K}$, based on epitaxial $\mathrm{HgCdTe}$ multi-layer-band gap engineered architecture.

\section{Simulation procedure and experimental details}

Numerical modelling of variable band gap and doping profile $\mathrm{HgCdTe}$ hetero-structures was based on the continuity equations for electrons and holes, Poisson and thermal conductivity equations $[7,8]$. The following mechanisms of dark current generation were considered: the Shockley-Read-Hall (SHR) and Auger 7 (Auger) generation-recombination processes, bandto-band (BTB), trap-assisted tunnelling (TAT) and dislocations-related generation (Dis).

Densities of dislocations $D_{D}=5 \times 10^{6} \mathrm{~cm}^{-2}$ and traps $N t=1 \times 10^{14} \mathrm{~cm}^{-3}$ were assumed to fit to the experimental results. A detailed description of calculation methods is given in $[9,10]$. The simulation results were used to select promising architectures, characterized by a low dark current, a high responsivity and minimized parasitic impedances.

The mid-IR HgCdTe photodiodes were fabricated in a joint laboratory run by VIGO System and Military University of Technology, Warsaw. The multilayer detector hetero-structures were grown on 2" semi-insulating, 2 deg disoriented (100) GaAs substrates in a horizontal MOCVD AIX 200 reactor. The inter-diffused multilayer process (IMP) technique was applied to the $\mathrm{HgCdTe}$ layer deposition. A detailed description of the implemented MOCVD growth procedure is presented in [12].

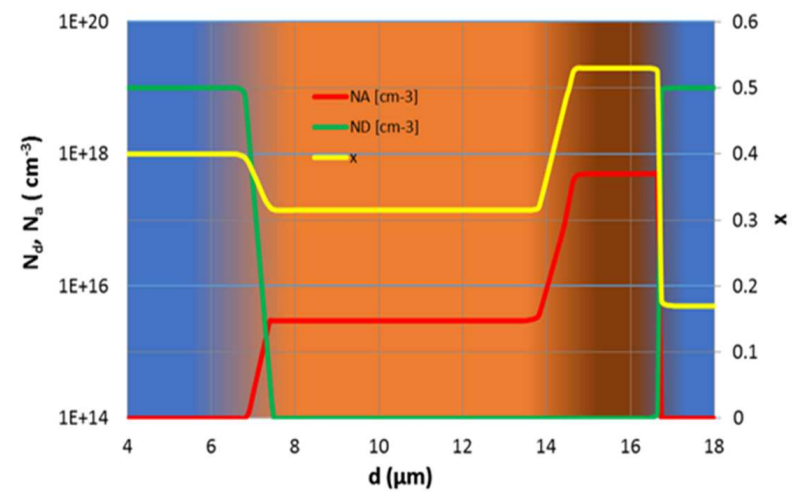

Fig. 1. A composition and doping profiles of the simulated $\mathrm{HgCdTe}$ hetero-structure.

An example of the photodiode structure is shown in Fig.1. The device consists of a $p$-type absorber, heavily doped wide-gap $\mathrm{P}^{+}$and $\mathrm{N}^{+}$hole and electron contacts and a heavily doped $\mathrm{n}^{+}$ layer used to minimize the series resistance between $\mathrm{P}^{+}$layer and contact metallization. The four main layers are separated by interfaces with graded gap and doping profiles. The composition of the device is obtained by a programmed change of growth temperature, precursor fluxes, mercury pressure, duration of IMP stages and other growth parameters [5]. It should be noted that the $\mathrm{CdTe} / \mathrm{HgTe}$ inter-diffusion and dopant diffusion processes were found to be slow for (100) growth compared with that for (111) orientation, so the interfaces were intentionally smoothed to prevent formation of dislocations by a suitable control of precursor fluxes.

The photodiodes were examined by measurements of a spectral responsivity, current voltage plots and a noise current. The time response was measured using an optical parametric oscillator (OPO) as the source of $\sim 25 \mathrm{ps}$ long, tuneable wavelength $(1.55-16 \mu \mathrm{m})$ pulses of IR radiation, a broad-bandwidth preamplifier and an $8 \mathrm{GHz}$ digital oscilloscope[12]. 


\section{Results and discussion}

Figure 2 shows simulated band structures. For the reverse bias electrons and holes are extracted from the absorber to $\mathrm{N}^{+}$and $\mathrm{P}^{+}$contacts, respectively. The carriers cannot be replenished by injection of minority carriers from the contact layer since their concentration there is very low. As a result, the absorber electrons are fully depleted while the hole concentration decreases to the extrinsic concentration, leading to suppression of Auger generation. To minimize the tunnel and generation-recombination in space charge regions, the device structure was designed for flat absorber bands and low electric fields (Fig. 2c) in the narrow gap part of absorber interfaces. In contrast, the $\mathrm{P}+\mathrm{n}+$ interface is made as sharp as possible to produce a low-resistance tunnelling contact.

Due to the flat band condition, the collection of photo-generated charge carriers mostly refers to diffusion rather than drift transport. The ambipolar diffusion coefficient in the depleted $\mathrm{p}$ type absorber is large, making the collection fast.

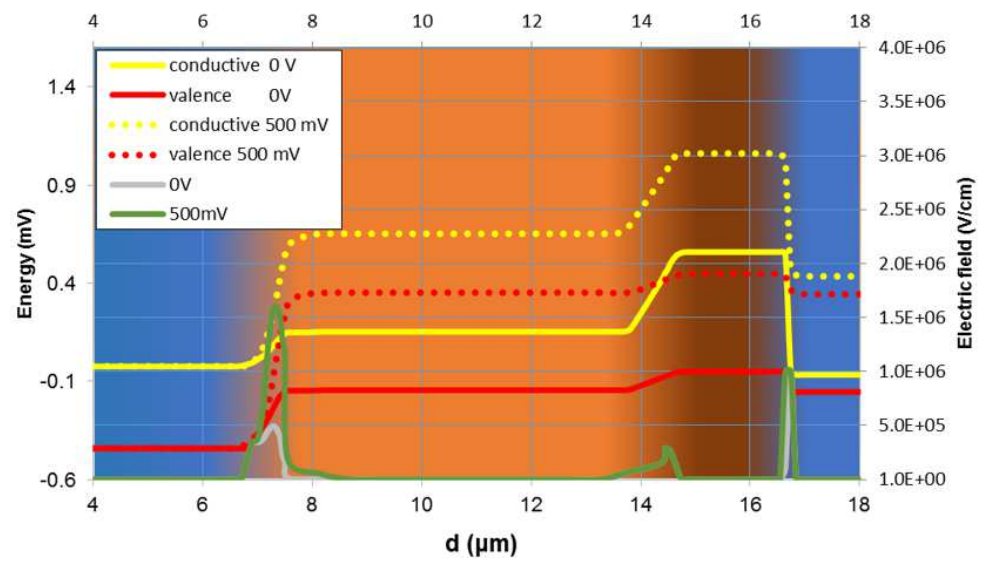

Fig. 2. The simulated band structure for $0 \mathrm{~V}$ and $500 \mathrm{mV}$ (yellow and red lines) bias voltages and electric fields of $0 \mathrm{~V}$ (grey line) and $500 \mathrm{mV}$ (green line).

Figure $3 \mathrm{c}$ shows the measured and calculated dark current-voltage plots. For the calculated dark current components - the Auger, SRH and Dis-related processes are shown. The first two mechanisms generate charge carriers mostly in the absorber region (Fig. $3 \mathrm{a}$ and $3 \mathrm{~b}$ ). The carriers diffuse to the contact regions resulting in a diffusion current which saturates at a reverse bias $>50 \mathrm{mV}$. It is worth mentioning that, in contrast to the homo-junction pin photodiodes, the SRH generation at absorber interfaces was to a large degree suppressed by an increased band gap.

The generation rate of Auger is large in the heavily doped $\mathrm{n}^{+}$contact region, but the generation does not produce a dark current due to the high electron barrier in $\mathrm{P}^{+} \mathrm{n}^{+}$interface.

In contrast, the dislocations-related current is generated mostly at interfaces with an increased dislocation density. The current increases with the bias voltage and dominates for large $(>700 \mathrm{mV})$ bias values.

Figure 4 shows the measured spectral responsivity and detectivity of optically immersed $\mathrm{HgCdTe}$ photodiode. The device is characterized by peak and cut-off wavelengths equal to 3.4 and $4.1 \mu \mathrm{m}$, respectively. The spectral current response remains nearly constant over a spectral range of 2.9 to $3.7 \mu \mathrm{m}$, with a high $\mathrm{R}_{\mathrm{i}} \sim 3 \mathrm{~A} / \mathrm{W}$ and $\mathrm{D}^{*} \sim 1.2 \cdot 10^{12} \mathrm{cmHz}^{1 / 2} / \mathrm{W}$. It should be noted that the measured detectivity of the device is larger compared with the BLIP $\left(300 \mathrm{~K}, 180^{\circ}\right)$ 
detectivity. This is due to the $36^{\circ}$ acceptance angle, limited by the hyper-hemispherical immersion lens.

a)

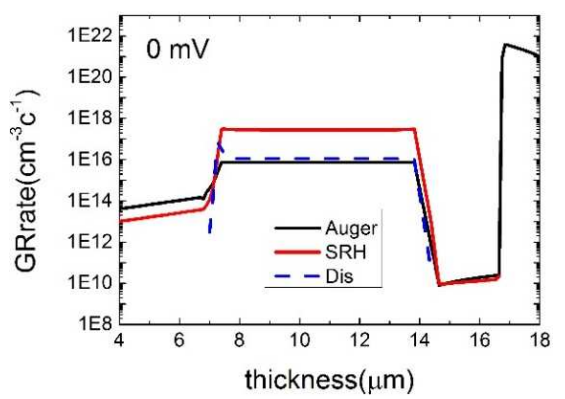

b)

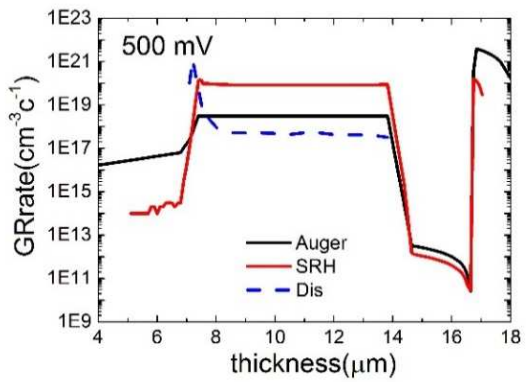

c)

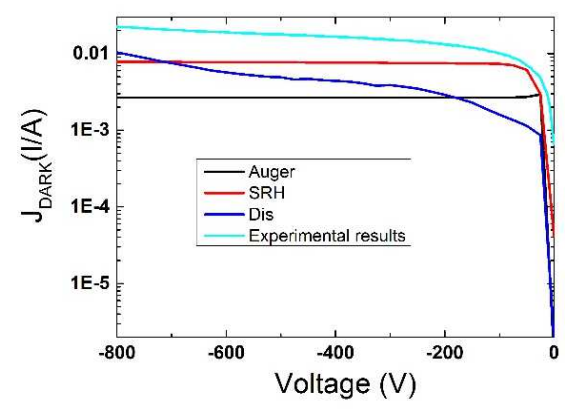

Fig. 3. The calculated and measured dark currents: the distribution of calculated thermal generation rates along the sample for Auger, SRH and Dis mechanisms for bias voltages of $0 \mathrm{mV}$ and $500 \mathrm{mV}$ (a, b); respectively the calculated Auger, SRH, Dis and the experimental results of thermal generation rates in a function of bias voltage (c).

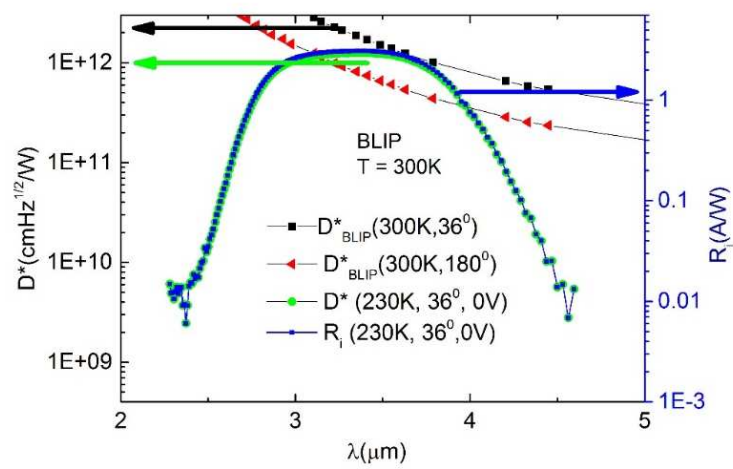

Fig. 4. The measured spectral current responsivity (blue curve), detectivity of the measured photodetector (green curve) and $\mathrm{D}_{\text {BLIP }}$ (black and red curve).

Figure 5 shows the simulated and measured response time as a function of bias voltage. The response is quite slow in unbiased devices and voltage signal readout conditions. This is due to a large diffusion capacitance for zero or small bias voltages, resulting in a large RC time constant. 
The response highly improves with increasing the bias voltage and the current mode readout. Picosecond-range time constants are easily achievable for small area devices operating with low $(<50 \mathrm{ohms})$ input resistance amplifiers.

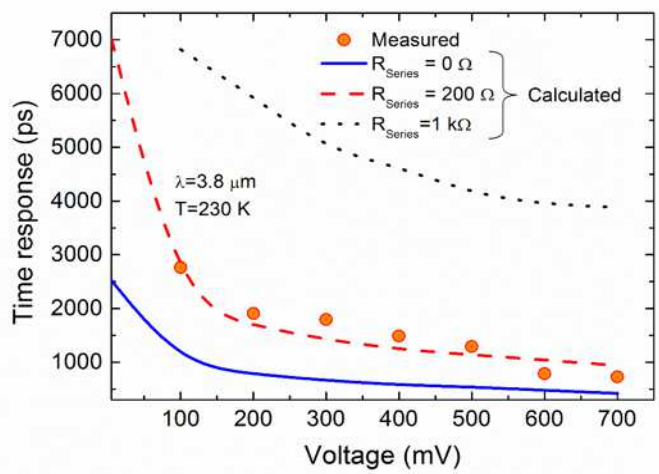

Fig. 5. The simulated and measured response time as a function of bias voltage

\section{Summary and conclusions}

Fast response HOT MWIR optically immersed $4.1 \mu \mathrm{m}$ cutoff photodiodes operating at $230 \mathrm{~K}$ have been designed by computer simulation of modified $\mathrm{N}^{+} \mathrm{pP}^{+} \mathrm{HgCdTe}$ heterostructures. The devices were implemented by MOCVD growth on GaAs substrate and examined by the current-voltage, noise and spectral measurements. Comparison of the calculated and measured J-V characteristics revealed the Auger and SRH mechanisms in the absorber to be responsible for the diffusion component of dark current, dominating at a weak $(<0.3 \mathrm{~V})$ reverse bias. Another important mechanism, significant at $>0.3 \mathrm{~V}$ and dominating at $>0.7 \mathrm{~V}$ bias, is the dislocation-related generation in absorber interfaces.

The optically immersed devices are characterized by a high $\left(1 \cdot 10^{12} \mathrm{cmHz}^{1 / 2} / \mathrm{W}\right.$ for $3 \mu \mathrm{m}<$ $\lambda<3.7 \mu \mathrm{m}$ ) detectivity, exceeding the $300 \mathrm{~K}, 180^{\circ}$ BLIP limit. This was possible due to reduction of the device acceptance angle to $\sim 36^{\circ}$ by the hyper-hemispherical immersion lenses.

Measurements of the time response using OPO sources of a short radiation pulse revealed its strong dependence on the detector bias and the type of signal readout. The response is quite slow for an unbiased device and/or voltage mode signal readout. This is due to a large RC time constant, determined by the product of the diffusion and depletion layer capacitance of the load and the detector series resistance. The time response highly improves with a reverse bias and a low resistance of signal readout electronics, that makes possible to obtain a picosecond-range response, eventually limited by the detector series resistance.

\section{Acknowledgments:}

This work was supported by Polish National Science Centre in the frame of the project NO. UMO-2013/08/A/ST5/00773

\section{References}

[1] Piotrowski, J., Rogalski, A. (2007). High-Operating-Temperature Infrared Photodetectors. SPIE, Bellingham. 
[2] Piotrowski, J., Galus, M., Grudzien, M. (1991). Near room-temperature IR photo-detectors. Infrared Phys., $31(1), 1-48$.

[3] Hunter, N., Mayorov, A.S., Wood, C.D., Russell, C., Li, L., Linfield, E.H., Davies, A.G., Cunningham, J.E. (2015). On-Chip Picosecond Pulse Detection and Generation Using Graphene Photoconductive Switches. Nano Lett., 15(3), 1591-1596.

[4] Newman, A.K., Liu, J.M. (1997). Physical characteristics of band-gap engineered, photovoltaic detectors. Journal of Applied Physics, 82(9), 4637-4646.

[5] Piotrowski, J., Gawron, W., Orman, Z., Pawluczyk, J., Kłos, K., Stępień, D., Piotrowski, A. (2010). Dark currents, responsivity, and response time in graded gap HgCdTe structures. Proc. SPIE, 7660, 766031.

[6] Spears, D.L. (1984). 10.6 micron photomixer arrays at 195. Proc. IRIS Active Systems, 331-349.

[7] Van Roosbroeck, W. (1950). Theory of the flow of electrons and holes in germanium and other semiconductors. Bell Syst. Tech. J., 29(4), 560-607.

[8] Kurata, M. (1982). Numerical Analysis for Semiconductor Devices. Lexington Books.

[9] Kopytko, M., Jóźwikowski, K., Jóźwikowska, A., Rogalski, A. (2010). High frequency response of nearroom temperature LWIR HgCdTe heterostructure photodiodes. Opto-Electronics Rev., 18(3), 277-283.

[10] Jóźwikowski, K., Jóźwikowska, A., Kopytko, M., Rogalski, A., Jaroszewicz, L.R. (2012). Simplified model of dislocations as a SRH recombination channel in the HgCdTe heterostructures. Infrared Physics \& Technology, 55(1), 98-107.

[11] Piotrowski, A., Madejczyk, P., Gawron, W., Kłos, K., Pawluczyk, J., Rutkowski, J., Piotrowski, J., Rogalski, A. (2007). Progress in MOCVD growth of HgCdTe heterostructures for uncooled infrared photodetectors. Infrared Phys. Technol., 49(3), 173-326

[12] Stanaszek, D., Piotrowski, J., Piotrowski, A., Gawron, W., Orman, Z., Paliwoda, R., Brudnowski, M., Pawluczyk, J., Pedzinska, M. (2009). Mid and long infrared detection modules for picosecond range measurements. Proc. SPIE, 7482, 74820, M-74820, M-11. 